\title{
Non-isothermal flow in low permeable porous media: A comparison of Richards' and two-phase flow approaches
}

\author{
Wenqing Wang · Jonny Rutqvist . \\ Uwe-Jens Görke · Jens T. Birkholzer · Olaf Kolditz
}

Received: March 15, 2010/ Accepted: date

\begin{abstract}
The present work compares the performance of two alternative flow models for the simulation of thermal-hydraulic coupled processes in low permeable porous media: non-
\end{abstract}

W. Wang · U.-J. Görke $(\bowtie) \cdot$ O. Kolditz

Helmholtz Centre for Environmental Research - UFZ

Permoserstr. 15, D-04318 Leipzig, Germany

E-mail: wenqing.wang@ufz.de

U.-J. Görke

Fon: $+49(0) 3412351804$

Fax: +49(0)3412351939

E-mail: uwe-jens.goerke@ufz.de

O. Kolditz

E-mail: olaf.kolditz@ufz.de

J. Rutqvist · J.T. Birkholzer

Lawrence Berkeley National Laboratory, Berkeley, USA

E-mail: jrutqvist@lbl.gov

J.T. Birkholzer

E-mail: JTBirkholzer@lbl.gov 
isothermal Richards' and two-phase flow concepts. Both models take vaporization processes

into account: however, the Richards' model neglects dynamic pressure variations and bulk flow of the gaseous phase. For the comparison of the two approaches first published data from a laboratory experiment is studied involving thermally driven moisture flow in a partially saturated bentonite sample. Then a benchmark test of longer-term thermal-hydraulic behavior in the engineered barrier system of a geological nuclear waste repository is analyzed (DECOVALEX project). It was found that both models can be used to reproduce the vaporization process if the intrinsic permeability is relative high. However, when a thermalhydraulic coupled problem has the same low intrinsic permeability for both the liquid and the gas phase, only the two-phase flow approach provides reasonable results.

Keywords Non-isothermal two-phase flow · Richards' approximation · Porous media · CTF1 experiment $\cdot$ DECOVALEX task D

\section{Introduction}

Performing numerical analyses of complex environmental problems and other real world applications, approximations of the governing equations are usually adopted according to specific features of the problem in order to gain an inexpensive computation without losing too much accuracy of the numerical solutions. One example of such an approximation is when less changeable state variables are neglected. Modeling of the thermal-hydro-mechanical (THM) coupled processes in low permeable porous media is a typical problem often requiring such simplifications. Low permeable bentonite-sand mixtures are frequently considered in geological nuclear waste repositories for tunnel back-fill and as an isolating buffer material embedding the disposed spent nuclear fuel assemblies. As a buffer in the Engineered Barrier System (EBS) of a geological nuclear waste repository, the bentonite-sand mixture 
is initially compacted to a tight, partially saturated porous medium of low permeability. The

bentonite buffer swells due to wetting from water intrusion and is subjected to significant changes in temperature conditions during long-term operation of the repository (Alkan and Müller 2008; Börgesson 1985; Börgesson et al. 2001; Chijimatsu et al. 2009; Gens and Alonso 1992).

To numerically simulate the THM processes associated with bentonite-sand-backfilled repositories, a sophisticated mathematical description of the coupled processes is essential, taking into account non-isothermal two-phase (gas and liquid) flow (Tsang 1991). However, most of the existing models for the analysis of coupled THM processes in partially saturated geological media are based on the Richards' approach and have frequently been applied to model non-isothermal fluid flow processes associated with back-filled nuclear waste repositories (Nguyen et al. 2009; Rutqvist et al. 2008, 2009). In the Richards' approach the gas phase is considered as a passive spectator under constant atmospheric pressure with no bulk gas flow. Vapor can still move within the static gas phase in the form of vapor diffusion caused by the vapor density gradient. The vapor gradient, in turn, depends on the gradients in temperature and capillary pressure.

Typically, some of the THM input parameters are determined by model calibration against small-scale laboratory experiments (Börgesson et al. 2001; Chijimatsu et al. 2009). Using such laboratory determined or calibrated input parameters, the Richards' approach has also been applied to model large-scale, multi-year in situ heating experiments, including the FEBEX in situ test at Grimsel, Switzerland (Alonso et al. 2005), and the Tunnel Sealing Experiment in the URL, Canada (Gou and Dixon 2006). A good agreement between observed temperature and saturation evolution indicates that the Richards' approach may be adequate for simulating THM processes in these kinds of systems. However, the good agreement may be a result of the fact that the model simulations were carried out with some 
input parameters calibrated using the same Richards' approach simplification, and doesn't

therefore constitute conclusive evidence that a full two-phase flow approach may not be necessary. In particular, the properties dictating the moisture transport under a thermal gradient have to be determined through a delicate model calibration against experiments that involve simultaneous liquid flow and vapor flow along opposite capillary and temperature gradients (Börgesson et al. 2001; Chijimatsu et al. 2009).

Thermo-hydro-mechanical (THM) modeling is an important analysis tool to other related environmental disciplines such as geothermal reservoir engineering (Bruel 2002; Kohl et al. 1995; Kolditz and Diersch 1993; Kuhn et al. 2002; O’Sullivan et al. 2001), $\mathrm{CO}_{2}$ sequestration ( $\mathrm{Li}$ et al. 2006; Pruess 2008), applications in soil mechanics (Freiboth et al. 2009; Zhang et al. 2009), and soil remediation (Jang and Aral 2009). Efficient solution of THM coupled problems requires the use of parallel computing (Schrefler et al. 2000; Wang et al. 2009).

The main goal of this paper is to systematically investigate the applicability of the Richards' approach and to compare this simplification with full two-phase flow under nonisothermal conditions corresponding to the EBS of a nuclear waste repository. According to the work by Olivella and Gens (2000), neglecting the gas phase flow in the fluid flow equation for TH coupled processes may cause vapor flux enhancement due to air immobility. Based on this fact, the purpose of this study is to further investigate the results of the two alternative flow approaches - non-isothermal two-phase flow model and the non-isothermal Richards' equation - for low permeable porous media. The finite element method within the framework of the OpenGeoSys code is utilized to discretize the weak forms of the two non-isothermal flow models. First, a laboratory test carried out at CIEMAT laboratories in Spain (Olivella and Gens 2000; Villar et al. 1997) is analyzed to study moisture flow processes under a thermal gradient. The saturation, temperature, and permeability responses 
are calculated numerically, and compared with measured profiles of saturation and temper-

ature along the sample. In particular, the paper analyzes at which conditions the Richards' simplified approach provides an admissible approximation. Finally, the applicability of the Richards' approach is investigated for the case of long-term thermal-hydraulic processes expected to occur at a generic back-filled nuclear waste repository. This is done by running a benchmark test comparing the results to published numerical data (Rutqvist et al. 2009, DECOVALEX project).

\section{Theoretical background}

As suggested by Sanavia et al. (2006), capillary pressure $p^{c}$, gas pressure $p^{g}$, and temperature $T$ are chosen as primary variables to establish the governing equations for nonisothermal two-phase flow in porous media. The solid skeleton of the porous medium under consideration is assumed to be undeformable.

\subsection{Mass balance equations}

Regarding the mass balance equation for complex fluid flow in porous media, the nonisothermal two-phase flow equations and their simplified form, the Richards' equation are considered.

\subsubsection{Non-isothermal two-phase flow}

For the presentation of the specific governing equations of interest, initially the general case of non-isothermal compositional two-phase flow in a porous medium is considered. Within this context, the model accounts for a significant coupling between flow and heat transport processes by temperature-dependent fluid properties and by coupling terms. The 
fluid mass balance equations under non-isothermal conditions can be derived from the mass conservation law (Kolditz and De Jonge 2004; Lewis and Schrefler 1998; Rutqvist et al. 2001; Sanavia et al. 2006). The component form (i.e. summed over phases) of the fluid mass balance equation is

$$
\frac{\partial}{\partial t}\left(n S^{g} \rho_{k}^{g}+n S^{l} \rho_{k}^{l}\right)+\nabla \cdot\left(\mathbf{J}_{k}^{g}+\mathbf{J}_{k}^{l}\right)=Q_{k}
$$

where the subscript $k$ denotes the components of the fluid, e.g. dry air $(k=a)$ and water ( $k=w$ ), $S$ is saturation, $n$ is the porosity, $\mathbf{J}$ is total flux, and $Q_{k}$ is the source/sink term.

For any phase $\gamma=(g, l)$, the density-like variables $\rho_{k}^{\gamma}$ denote the intrinsic phase-averaged mass concentrations of the respective component in the corresponding phase of interest. An advection vector $\mathbf{J}_{A}^{\gamma}$ and a diffusion vector $\mathbf{J}_{D_{k}}^{\gamma}$ constitute the particular total flux, i.e.

$$
\mathbf{J}_{k}^{\gamma}=\mathbf{J}_{A_{k}}^{\gamma}+\mathbf{J}_{D_{k}}^{\gamma}
$$

According to the Darcy equation, the advective part of the total flux can be written as

$$
\mathbf{J}_{A}^{\gamma}=-\rho_{k}^{\gamma} \frac{\mathbf{k} k_{\mathrm{rel}}^{\gamma}}{\mu^{\gamma}}\left(\nabla p^{\gamma}-\rho^{\gamma} \mathbf{g}\right)
$$

where $\mathbf{k}$ is the intrinsic permeability, $k_{\text {rel }}^{\gamma}$ is the relative permeability, $\mu^{\gamma}$ is the viscosity, $p^{\gamma}$ is the pressure, $\rho^{\gamma}$ is the intrinsic phase-averaged density of the respective fluid phase $\gamma$, and $\mathbf{g}$ is the gravity vector. Analogously, using Fick's law, the diffusion part of the total flux vector can be defined as

$$
\mathbf{J}_{D_{k}}^{\gamma}=-\rho^{\gamma} \mathbb{D}_{k}^{\gamma} \nabla\left(\frac{\rho_{k}^{\gamma}}{\rho^{\gamma}}\right)
$$

where $\mathbb{D}_{k}^{\gamma}$ is the diffusion tensor.

Within the context of phase changes, in the following only vaporization will be considered, whereas effects on the liquid phase due to condensation processes are neglected. Consequently, the gas phase is assumed to be constituted of dry air and water vapor, and the 
liquid phase consists solely of the water component. Based on these assumptions, diffusion is observed only in the binary gas phase, and the corresponding diffusion parts of the total flux vectors can be obtained as

$$
\mathbf{J}_{D_{k}^{g}}^{g}=-\rho^{g} \frac{M_{a} M_{w}}{M_{g}^{2}} \mathbb{D}_{k}^{g} \nabla\left(\frac{p_{k}^{g}}{p^{g}}\right), \quad \mathbf{J}_{D k}^{l}=\mathbf{0}
$$

(cf. Lewis and Schrefler 1998, and others). Here, $p_{k}^{g}$ denotes the partial pressure induced by phase change, $M_{a}$ is the molar mass of dry air, $M_{w}$ is the molar mass of water, and $M_{g}$ is the molar mass of the gas phase mixture given by

$$
\frac{1}{M_{g}}=\frac{1}{\rho^{g}}\left(\frac{\rho_{w}^{g}}{M_{w}}+\frac{\rho_{a}^{g}}{M_{a}}\right)
$$

with the density of the gas phase mixture $\rho^{g}$, the mass concentration of dry air component $\rho_{a}^{g}$, and the mass concentration of water vapor component $\rho_{w}^{g}$ in the gas phase. Since $p^{g}=$ $p_{a}^{g}+p_{w}^{g}$, for the binary gas phase holds

$$
\mathbf{J}_{D}^{g}+\mathbf{J}_{D a}^{g}=\mathbf{0}
$$

under the physically appropriate assumption of $\mathbb{D}_{a}^{g}=\mathbb{D}_{w}^{g}:=\mathbb{D}^{g}$.

Considering the appropriate definition of flux vectors (2), (3) and (5), the mass balance equation for water and water vapor can be written as follows

$$
\begin{gathered}
\frac{\partial}{\partial t}\left(n S^{g} \rho_{w}^{g}+n S^{l} \rho_{w}^{l}\right)-\nabla \cdot\left[\rho_{w}^{l} \frac{\mathbf{k} k_{\mathrm{rel}}^{l}}{\mu^{l}}\left(\nabla p^{l}-\rho^{l} \mathbf{g}\right)\right] \\
-\nabla \cdot\left[\rho_{w}^{g} \frac{\mathbf{k} k_{\mathrm{rel}}^{g}}{\mu^{g}}\left(\nabla p^{g}-\rho^{g} \mathbf{g}\right)\right] \\
-\nabla \cdot\left[\rho^{g} \frac{M_{a} M_{w}}{M_{g}^{2}} \mathbb{D}^{g} \nabla\left(\frac{p_{w}^{g}}{p^{g}}\right)\right]=Q_{w}
\end{gathered}
$$


Since the capillary pressure $p^{c}:=p^{g}-p^{l}$ is chosen as a primary variable instead of saturation $S$ of equation (1) and $S^{g}=1-S^{l}$, equation (8) becomes

$$
\begin{aligned}
n\left(\rho_{w}^{l}\right. & \left.-\rho_{w}^{g}\right)\left(\frac{\partial S^{l}}{\partial T} \frac{\partial T}{\partial t}+\frac{\partial S^{l}}{\partial p^{c}} \frac{\partial p^{c}}{\partial t}\right) \\
& +\left(1-S^{l}\right) n\left(\frac{\partial \rho_{w}^{g}}{\partial T} \frac{\partial T}{\partial t}+\frac{\partial \rho_{w}^{g}}{\partial p^{g}} \frac{\partial p^{g}}{\partial t}+\frac{\partial \rho_{w}^{g}}{\partial p^{c}} \frac{\partial p^{c}}{\partial t}\right) \\
& -\nabla \cdot\left[\rho_{w}^{l} \frac{\mathbf{k} k_{\mathrm{rel}}^{l}}{\mu^{l}}\left(\nabla\left(p^{g}-p^{c}\right)-\rho^{l} \mathbf{g}\right)\right] \\
& -\nabla \cdot\left[\rho_{w}^{g} \frac{\mathbf{k} k_{\mathrm{rel}}^{g}}{\mu^{g}}\left(\nabla p^{g}-\rho^{g} \mathbf{g}\right)\right] \\
- & {\left[\rho^{g} \frac{M_{a} M_{w}}{M_{g}^{2}} \mathbb{D}^{g} \nabla\left(\frac{p_{w}^{g}}{p^{g}}\right)\right]=Q_{w} }
\end{aligned}
$$

Within this context, two further assumptions are used: Due to the rigid behavior of the solid skeleton, the porosity does not change, and the liquid water is assumed to be incompressible.

Following the same procedure as for the mass balance equation for water and water vapor, the mass balance equation for the dry air component is derived as:

$$
\begin{gathered}
\frac{\partial}{\partial t}\left(n S^{g} \rho_{a}^{g}\right)-\nabla \cdot\left[\rho_{a}^{g} \frac{\mathbf{k} k_{\mathrm{rel}}^{g}}{\mu^{g}}\left(\nabla p^{g}-\rho^{g} \mathbf{g}\right)\right] \\
-\nabla \cdot\left[\rho^{g} \frac{M_{a} M_{w}}{M_{g}^{2}} \mathbb{D}^{g} \nabla\left(\frac{p_{a}^{g}}{p^{g}}\right)\right]=Q_{a}
\end{gathered}
$$

Analyzing the time derivative term of equation (10) within the context of dependences on capillary pressure and temperature as primary variables yields

$$
\begin{aligned}
-n \rho_{a}^{g} & \left(\frac{\partial S^{l}}{\partial T} \frac{\partial T}{\partial t}+\frac{\partial S^{l}}{\partial p^{c}} \frac{\partial p^{c}}{\partial t}\right) \\
& +\left(1-S^{l}\right) n\left(\frac{\partial \rho_{a}^{g}}{\partial T} \frac{\partial T}{\partial t}+\frac{\partial \rho_{a}^{g}}{\partial p^{g}} \frac{\partial p^{g}}{\partial t}+\frac{\partial \rho_{a}^{g}}{\partial p^{c}} \frac{\partial p^{c}}{\partial t}\right) \\
& -\nabla \cdot\left[\rho_{a}^{g} \frac{\mathbf{k} k_{\mathrm{rel}}^{g}}{\mu^{g}}\left(\nabla p^{g}-\rho^{g} \mathbf{g}\right)\right] \\
- & \nabla \cdot\left[\rho^{g} \frac{M_{a} M_{w}}{M_{g}^{2}} \mathbb{D}^{g} \nabla\left(\frac{p_{a}^{g}}{p^{g}}\right)\right]=Q_{a}
\end{aligned}
$$


According to the Clapeyron equation for an ideal gas and Dalton's law holds

$$
\begin{aligned}
& p_{a}^{g}=\rho_{a}^{g} R T / M_{a}, p_{w}^{g}=\rho_{w}^{g} R T / M_{w} \\
& p^{g}=p_{a}^{g}+p_{w}^{g}, \quad \rho^{g}=\rho_{a}^{g}+\rho_{w}^{g}
\end{aligned}
$$

where $R$ is the universal gas constant with a value of $8.314 \mathrm{~J} /(\mathrm{mol} \cdot \mathrm{K})$. In the unsaturated zone, the equilibrium water vapor pressure is given by the Kelvin-Laplace equation

$$
p_{w}^{g}=p_{w s}^{g} \exp \left(-\frac{p^{c} M_{w}}{\rho_{w}^{l} R T}\right)
$$

Thereby, an empirical water vapor saturation function $p_{w s}^{g}$ is used, as given in (Philip and de Vries 1957)

$$
p_{w s}^{g}=10^{-3} \exp (19.84-4975.9 / T) \frac{R T}{M_{w}}
$$

With the constitutive equations (12), (13) and (14), the gas phase density related derivative terms of mass balance equations (9) and (11) are now dependent on the primary variable $p^{c}$. In order to enhance the numerical stability, the gas phase density related derivative terms are expanded by the chain rule as follows

$$
\begin{gathered}
\frac{\partial p^{g}}{\partial p^{c}}=0 \Longrightarrow \frac{\partial p_{a}^{g}}{\partial p^{c}}=-\frac{\partial p_{w}^{g}}{\partial p^{c}} \\
\nabla\left(\frac{p_{a}^{g}}{p^{g}}\right)=-\nabla\left(\frac{p_{w}^{g}}{p^{g}}\right) \\
=\frac{p_{w}^{g}}{\left(p^{g}\right)^{2}} \nabla p^{g}+\frac{\rho_{w}^{g}}{p^{g} \rho_{w}^{l}} \nabla p^{c}-\frac{1}{p^{g}} \frac{\partial p_{w}^{g}}{\partial T} \nabla T
\end{gathered}
$$

Formula (16) represents the spatial variation of the mass transition from liquid phase to gaseous phase, and it prompts that the thermal diffusion term contributes to the Laplacians on the left hand side of equations (9) and (11). 
The Richards' model neglects the mobility of the gas phase in the porous medium, i.e. gas pressure changes are assumed to be very small. With the assumption $p^{g}=$ const, the generic form of the mass balance equation for the first component, i.e. equation (9), can be represented as an expression with water pressure as primary variable. The equation for the second component (11) can be neglected. This yields a simplified pressure based mass balance equation as follows

$$
\begin{aligned}
n\left(\rho_{w}^{l}\right. & \left.-\rho_{w}^{g}\right)\left(\frac{\partial S^{l}}{\partial T} \frac{\partial T}{\partial t}-\frac{\partial S^{l}}{\partial p^{c}} \frac{\partial p^{l}}{\partial t}\right) \\
+ & \left(1-S^{l}\right) n\left(\frac{\partial \rho_{w}^{g}}{\partial T} \frac{\partial T}{\partial t}-\frac{\partial \rho_{w}^{g}}{\partial p^{c}} \frac{\partial p^{l}}{\partial t}\right) \\
& -\nabla \cdot\left[\rho_{w}^{l} \frac{\mathbf{k} k_{\mathrm{rel}}^{l}}{\mu^{l}}\left(\nabla p^{l}-\rho^{l} \mathbf{g}\right)\right] \\
- & -\left[\rho^{g} \frac{M_{a} M_{w}}{M_{g}^{2}} \mathbb{D}^{g} \nabla\left(\frac{p_{w}^{g}}{p^{g}}\right)\right]=Q_{w}
\end{aligned}
$$

Considering equation (16), the vapor flux term can be expressed as

$$
\begin{aligned}
& \rho^{g} \frac{M_{a} M_{w}}{M_{g}^{2}} \mathbb{D}^{g} \nabla\left(\frac{p_{w}^{g}}{p^{g}}\right) \\
& \quad \approx \rho^{g} \frac{M_{a} M_{w}}{M_{g}^{2}} \mathbb{D}^{g}\left(\frac{\rho_{w}^{g}}{p^{g} \rho_{w}^{l}} \nabla p^{l}+\frac{1}{p^{g}} \frac{\partial p_{w}^{g}}{\partial T} \nabla T\right)
\end{aligned}
$$

According to (Rutqvist et al. 2001), the coefficients on the right hand side of equation (18) can be further simplified as

$$
\rho^{g} \frac{M_{a} M_{w}}{M_{g}^{2}} \mathbb{D}^{g} \nabla\left(\frac{p_{w}^{g}}{p^{g}}\right) \approx D_{p v} \nabla p^{l}+f_{T v} D_{T v} \nabla T
$$


where $f_{T v}$ represents an enhancement factor for the thermal diffusion term. The variables $D_{p v}$ and $D_{T v}$ denote particular diffusion coefficients given by

$$
\begin{aligned}
D_{p v} & =\frac{D_{v} \rho_{w}^{g}}{\rho_{w}^{l} R_{v} T} \\
D_{T v} & =D_{v}\left(\theta \frac{\partial \rho_{v S}}{\partial T}-\frac{\rho_{w}^{g} p^{l}}{\rho_{w}^{l} R_{v} T^{2}}\right)
\end{aligned}
$$

with $R_{v}=461.5 \mathrm{~J} /(\mathrm{kg} \cdot \mathrm{K})$ the specific gas constant, $D_{v}$ the vapor diffusion coefficient, $\rho_{v S}$ the saturated vapor density, and $\theta=\exp \left(p^{l} / \rho_{w}^{l} R_{v} T\right)$ the relative humidity. The saturated vapor density is given by an empirical function (Rutqvist et al. 2001)

$$
\rho_{v S}=10^{-3} \exp (19.891-4975 / T)
$$

\subsection{Energy balance equation}

The heat transport equation for two-phase flow in porous media can be derived from the energy balance (Gray and Hassanizadeh 1991; Kolditz and De Jonge 2004). The coupling with fluid flow is represented by two convective transport terms (for liquid and gas) in the governing equation as follows

$$
\begin{aligned}
\left(\rho C_{p}\right)_{\mathrm{eff}} \frac{\partial T}{\partial t} & +\rho^{l} C_{p}^{l} \frac{\mathbf{k} k_{\mathrm{rel}}^{l}}{\mu^{l}}\left(-\nabla p^{g}+\nabla p^{c}+\rho^{l} \mathbf{g}\right) \nabla T \\
& +\rho^{g} C_{p}^{g} \frac{\mathbf{k} k_{\mathrm{rel}}^{g}}{\mu^{g}}\left(-\nabla p^{g}+\rho^{g} \mathbf{g}\right) \nabla T \\
& -\nabla \cdot\left(K_{\mathrm{e}} \nabla T\right)+Q_{\mathrm{T}}=0
\end{aligned}
$$

where $\rho$ is the density of the porous medium, $K_{\mathrm{e}}$ is the heat conductivity, and $Q_{\mathrm{T}}$ is the source term. The effective heat capacity is described by

$$
\left(\rho C_{p}\right)_{\mathrm{eff}}=(1-n) \rho^{s} C_{p}^{s}+n\left(S^{l} \rho^{l} C_{p}^{l}+S^{g} \rho^{g} C_{p}^{g}\right)
$$


where $C_{p}^{s}, C_{p}^{l}$ and $C_{p}^{g}$ denote the specific heat capacity of the solid phase, the liquid phase, and the gas phase, respectively.

For the Richards' model, the energy balance takes a simplified form of equation (22) as

$$
\begin{gathered}
\left(\rho C_{p}\right)_{\mathrm{eff}} \frac{\partial T}{\partial t}+\rho^{l} C_{p}^{l} \frac{\mathbf{k} k_{\mathrm{rel}}^{l}}{\mu^{w}}\left(-\nabla p^{l}+\rho^{l} \mathbf{g}\right) \nabla T \\
-\nabla \cdot\left(K_{\mathrm{e}} \nabla T\right)+Q_{\mathrm{T}}=0
\end{gathered}
$$

\subsection{Numerical scheme}

The method of weighted residuals is applied to derive the corresponding weak form of the balance equations. Spatially discretizing these weak formulations within the finite element space using the Galerkin procedure and applying a staggered scheme for the coupling terms which are contained in the equations (9), (11) and (22) leads to a system of ordinary differential equations with respect to time derivatives

$$
\begin{gathered}
\mathbf{M}_{p} \dot{\mathbf{p}}+\mathbf{K}_{p} \mathbf{p}=\mathbf{f}_{p} \\
\mathbf{M}_{T} \dot{\mathbf{T}}+\mathbf{K}_{T} \mathbf{T}=\mathbf{f}_{T}
\end{gathered}
$$

where the corresponding matrices and vectors for flow and heat transport terms are denoted by subscripts $p$ and $T$, respectively. The parameters $\mathbf{p}=\left(\mathbf{p}^{c}, \mathbf{p}^{g}\right)^{\operatorname{tr}}$ and $\mathbf{T}$ stand for fluid pressure unknowns and temperature unknowns at element nodes. The mass and Laplacian matrices are denoted by $\mathbf{M}$ and $\mathbf{K}$, respectively, and $\mathbf{f}$ is the right hand side vector. For the temporal discretization, the backward Euler or generalized implicit methods are applied. Considering a relaxation approach for the Laplacian terms, equation (25) can be discretized as follows

$$
\begin{aligned}
& \mathbf{M}_{p}\left(\mathbf{p}_{i+1}-\mathbf{p}_{i}\right) / \Delta t+\mathbf{K}_{p}\left[\alpha \mathbf{p}_{i+1}+(1-\alpha) \mathbf{p}_{i}\right]=\mathbf{f}_{p} \\
& \mathbf{M}_{T}\left(\mathbf{T}_{i+1}-\mathbf{T}_{i}\right) / \Delta t+\mathbf{K}_{T}\left[\alpha \mathbf{T}_{i+1}+(1-\alpha) \mathbf{T}_{i}\right]=\mathbf{f}_{T}
\end{aligned}
$$


where $i$ is the time step index, $\Delta t=t_{i+1}-t_{i}$ denotes time step size, and $\alpha \in[0,1]$ is a relaxation parameter.

The mass matrices $\mathbf{M}$, Laplacian matrices $\mathbf{K}$ and right hand sides $\mathbf{f}$ are functions of the primary nodal variables $\mathbf{p}_{i+1}=\left(\mathbf{p}_{c}, \mathbf{p}_{g}\right)_{i+1}^{\mathrm{tr}}$ or $\mathbf{T}_{i+1}$. Within this context, the fix point or the Picard method is adopted to linearize equation (26). Therefore, for each Picard iteration $\tau+1$, the solution of equation (26) is given by

$$
\begin{aligned}
& \mathbf{p}_{i+1}^{\tau+1}=\mathbf{H}_{p}^{\tau}\left(\mathbf{f}_{p}^{\tau}+\mathbf{M}_{p}^{\tau} \mathbf{p}_{i} / \Delta t-(1-\alpha) \mathbf{K}_{p}^{\tau} \mathbf{p}_{i}\right) \\
& \mathbf{T}_{i+1}^{\tau+1}=\mathbf{H}_{T}^{\tau}\left(\mathbf{f}_{T}^{\tau}+\mathbf{M}_{T}^{\tau} \mathbf{T}_{i} / \Delta t-(1-\alpha) \mathbf{K}_{T}^{\tau} \mathbf{T}_{i}\right)
\end{aligned}
$$

with

$$
\begin{aligned}
& \mathbf{H}_{p}^{\tau}=\left[\mathbf{M}_{p}^{\tau} / \Delta t+\alpha \mathbf{K}_{p}^{\tau}\right]^{-1} \\
& \mathbf{H}_{T}^{\tau}=\left[\mathbf{M}_{T}^{\tau} / \Delta t+\alpha \mathbf{K}_{T}^{\tau}\right]^{-1}
\end{aligned}
$$

where $\tau$ indicates the previous Picard iteration.

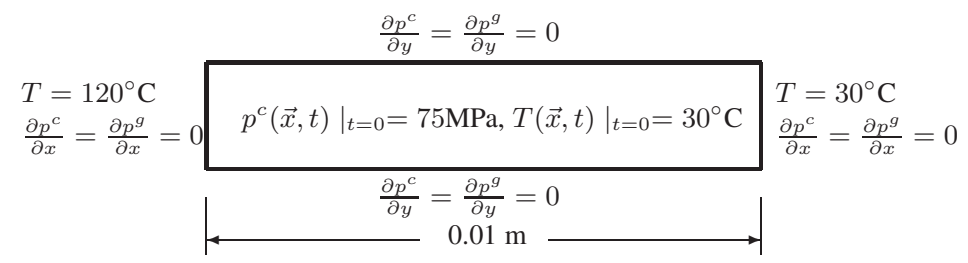

Fig. 1 Numerical model of the CTF1 experiment

\section{Simulations and comparison}

In this section both the Richards' and full two-phase flow model approaches are applied for the numerical simulation of a laboratory test conducted by Villar et al. (1997), and then for the simulation of a benchmark test involving longer-term $\mathrm{TH}$ processes around a backfilled nuclear waste repository (Barr et al. 2004, DECOVALEX project). 
Table 1 Parameters for the non-isothermal flow simulations of the CTF1 experiment (in parts given by

Olivella and Gens 2000)

\begin{tabular}{|c|c|c|c|}
\hline Parameter & Symbol & Value & Unit \\
\hline Porosity & $n_{0}$ & 0.44 & - \\
\hline Initial clay density & $\rho$ & 1.65 & $\mathrm{~kg} / \mathrm{m}^{3}$ \\
\hline Intrinsic permeability & $\mathbf{k}$ & Eqn. (29) & $\mathrm{m}^{2}$ \\
\hline Liquid viscosity & $\mu^{l}$ & $1.0 \times 10^{-3}$ & $\mathrm{~Pa} \cdot \mathrm{s}$ \\
\hline Air viscosity & $\mu^{g}$ & $1.8 \times 10^{-5}$ & $\mathrm{~Pa} \cdot \mathrm{s}$ \\
\hline Relative permeability of liquid & $k_{\mathrm{rel}}^{l}$ & $\left(S^{l}\right)^{12}$ & - \\
\hline Relative permeability of gas & $k_{\text {rel }}^{g}$ & $1-S^{l}$ & - \\
\hline Residual water saturation & $S_{r}^{l}$ & 0.0 & - \\
\hline Residual gas saturation & $S_{r}^{g}$ & 0.0 & - \\
\hline van Genuchten index & & 0.38 & - \\
\hline Entry pressure & & 18.0 & MPa \\
\hline Liquid density & $\rho^{l}$ & 1000.0 & $\mathrm{~kg} / \mathrm{m}^{3}$ \\
\hline Specific heat capacity of liquid phase & $C_{p}^{l}$ & $4.28 \times 10^{3}$ & $\mathrm{~J} /(\mathrm{kg} \cdot \mathrm{K})$ \\
\hline Specific heat capacity of gas phase & $C_{p}^{g}$ & $1.01 \times 10^{3}$ & $\mathrm{~J} /(\mathrm{kg} \cdot \mathrm{K})$ \\
\hline Specific heat capacity of solid phase & $C_{p}^{s}$ & $1.38 T+732.5$ & $\mathrm{~J} /(\mathrm{kg} \cdot \mathrm{K})$ \\
\hline Heat conductivity of porous medium & $K_{\mathrm{e}}$ & $0.5 S^{g}+1.28 S^{l}$ & $\mathrm{~W} /(\mathrm{m} \cdot \mathrm{K})$ \\
\hline
\end{tabular}

\subsection{CTF1 experiment}

In the CTF1 experiment carried out by Villar et al. (1997), a $10 \mathrm{~cm}$ clay sample is subjected to a temperature gradient of $9^{\circ} \mathrm{C} / \mathrm{cm}$. The material is partially saturated at the beginning.

Liquid and gas flow are suppressed through the boundaries of the specimen. A numerical 
model of the experiment was generated as illustrated in Fig. 1, which shows the selected initial and boundary conditions for flow and heat transport.

For the numerical simulation, the material parameters used by Olivella and Gens (2000) were adapted directly. Within this context, for both unsaturated and two-phase flow models, the same van Genuchten water retention curve (van Genuchten 1980) is applied. The material parameters are given in Table 1.

According to (Olivella and Gens 2000), the intrinsic permeability $\mathbf{k}$

$$
\mathbf{k}\left(S^{l}\right)=\mathbf{k}\left(n_{M}\right)=k_{0}\left(\frac{1-n_{0}}{1-n_{M}}\right)^{2}\left(\frac{n_{M}}{n_{0}}\right)^{3} \mathbf{I}
$$

with $n_{0}$ and $k_{0}$ reference values for porosity and intrinsic permeability, respectively, and $\beta$ a material constant, is a function of the macro porosity $n_{M}$, which is given by

$$
n_{M}=n_{0} e^{-\beta S^{l}}
$$

Relation (29) implies that intrinsic permeability varies with saturation to mimic the interaction between micro and macro porosity in swelling clay.

The column type experiment can be considered as one dimensional problem. Within the context of the spatial discretization, the domain is divided into 270 line elements. The simulations are carried out for the experiment with a total duration of 14 days. Both simulations corresponding to the Richards' and two-phase flow models are conducted under exactly the same parameterization as well as initial and boundary conditions.

According to the definition of the gradient of vapor mass fraction in gas, the low intrinsic permeability gives rise to a low vapor mass fraction gradient, and consequently leads to a small drying effect induced by the temperature gradient (cf. Olivella and Gens 2000). To investigate the behavior of the two non-isothermal flow models applied to the drying problems in porous media, the thermal and hydraulic coupled processes have been simulated 
in this experiment with three different values of the intrinsic permeability parameters of expression (29), which range from relative large ones, $k_{0}=10^{-11} \mathrm{~m}^{2}$ and $k_{0}=10^{-13} \mathrm{~m}^{2}$, to a very small one $k_{0}=10^{-18} \mathrm{~m}^{2}$. With each of the three selected $k_{0}$ values, several simulations are conducted varying the coefficient $\beta$ to best match the experiment data. The simulations results show that with the decrease of $k_{0}$, the value of $\beta$ should be decreased as well in order to achieve a better agreement between the simulation results and the experimental data. Corresponding to the three selected $k_{0}$ values of $10^{-11}, 10^{-13}$ and $10^{-18} \mathrm{~m}^{2}$, was found that $\beta=7, \beta=4.2$ and $\beta=0$ provide the best fit with the data.

\subsubsection{Results with high intrinsic permeability}

Firstly, results using the high intrinsic permeability values of $k_{0}=10^{-11} \mathrm{~m}^{2}$ and $k_{0}=$ $10^{-13} \mathrm{~m}^{2}$ are discussed within the context of the comparison of two non-isothermal numerical approaches: two-phase flow model and its Richards' approximation. The latter value, $k_{0}=10^{-13} \mathrm{~m}^{2}$, is the same as the one used in the work by Olivella and Gens (2000). In Fig. 2, the intrinsic permeability distribution in the domain at different times is plotted for cases of $k_{0}=10^{-11} \mathrm{~m}^{2}$ and $k_{0}=10^{-13} \mathrm{~m}^{2}$, respectively.

Fig. 2 shows that for the same values of intrinsic permeability parameters, the Richards' approach results in a slightly higher permeability on the heated side and a smaller permeability on the cool side compared with the two-phase approach.

From Fig. 3 can be seen that the saturation results obtained with two values of permeability, $k_{0}=10^{-11} \mathrm{~m}^{2}$ and $k_{0}=10^{-13} \mathrm{~m}^{2}$, are almost identical, and they both agree well with the experimental results presented by Olivella and Gens (2000). However, in the case of a lower permeability $\left(k_{0}=10^{-11} \mathrm{~m}^{2}, \beta=4.2\right)$, the drying is slightly stronger leading to a slightly lower saturation at the end of the 14-day experiment. 
Following, with the same two sets of values for the reference permeability, the CTF1 experiment is analyzed using the Richards' equation, taking the vaporization into account.

The comparison of the results obtained by the two flow models within the context of the saturation distribution is illustrated in Fig. 4.

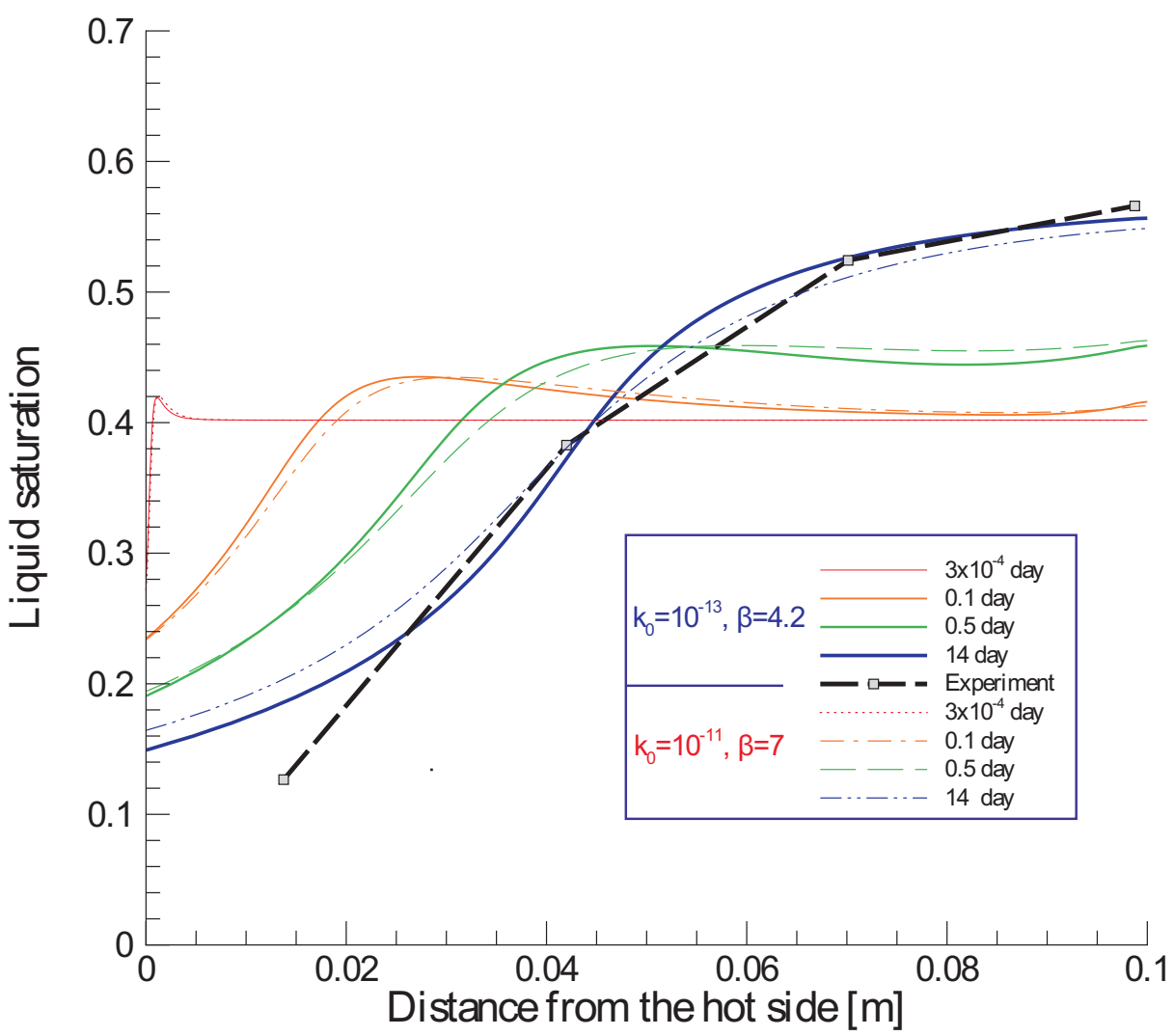

Fig. 3 Saturation profiles by two-phase flow simulations of CTF1 at different times $t=3 \times 10^{-4}, 0.1,0.5$, 14 days (experimental data is given for $t=14$ days) 
It can be clearly seen that vaporization occurs on the heated side, i.e., the saturations are decreasing with time. Both the unsaturated and two-phase flow model results at 14 days match the measured data well except in the vicinity of the heating boundary condition. The results in Fig. 4 indicate that in this type of problem the Richards' model results in a lower saturation on the heated side and a slightly higher saturation on the cold side. Overall, however, the saturation results by both flow models are quite close to each other. The simulated temperature profiles by both flow models are almost identical as shown in Fig. 5. However, after 14 days of heating, the two-phase flow approach shows a slightly stronger advection effect than the Richards' approach when the intrinsic permeability is lower (see Fig. 5a). The situation is reversed when the intrinsic permeability is higher (see Fig. 5b). 

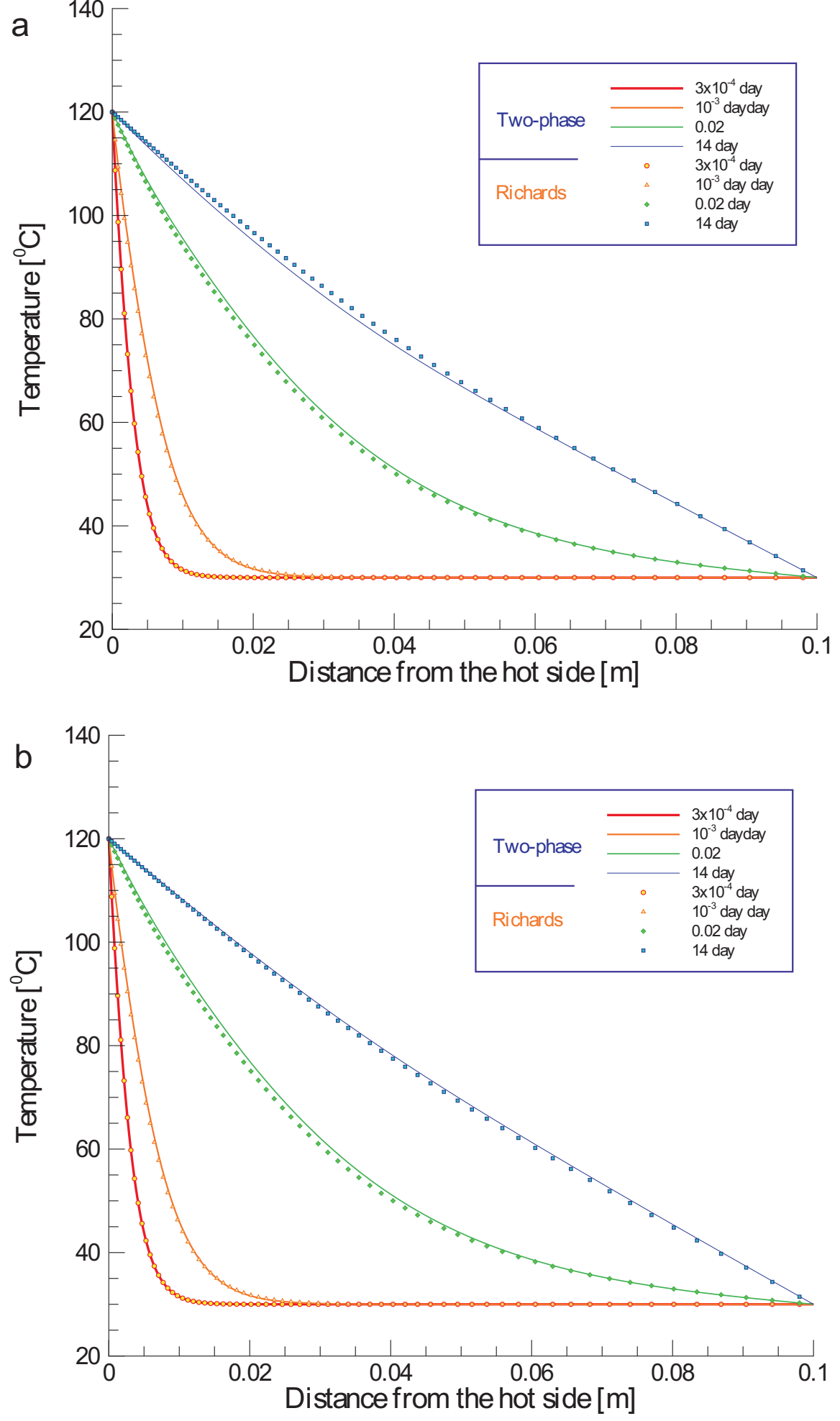

Fig. 5 Temperature profiles resulting from the simulations of CTF1 at different times $t=3 \times 10^{-4}, 0.1,0.5$, 14 days: a) $k=10^{-13} \mathrm{~m}^{2}, \beta=4.2$; b) $k=10^{-11} \mathrm{~m}^{2}, \beta=7$ 


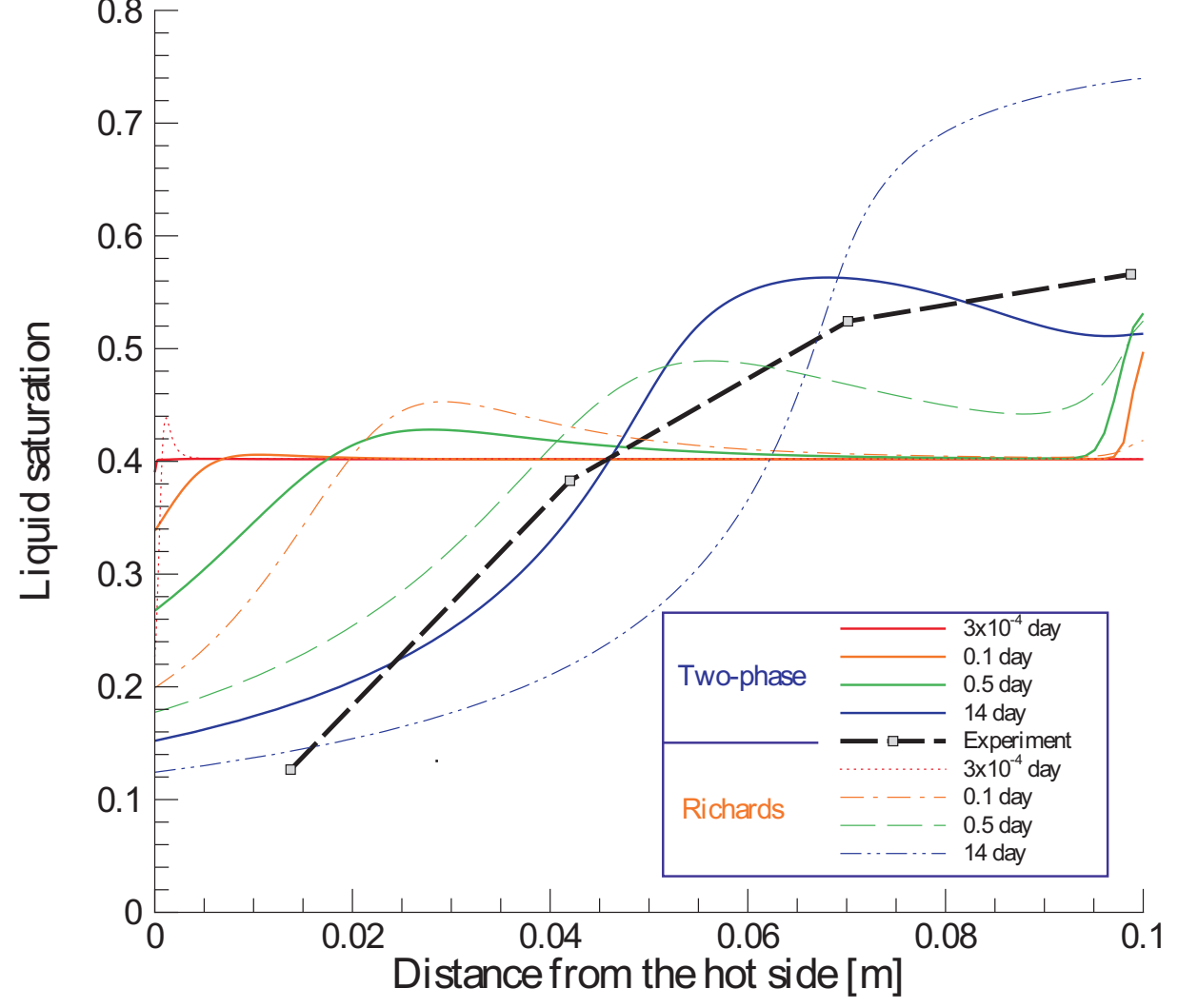

Fig. 6 Saturation profiles of CTF1 modeling with low intrinsic permeability at different times $t=3 \times 10^{-4}$, $0.1,0.5,14$ days (experimental data is given for $t=14$ days)

\subsubsection{Results with low intrinsic permeability}

As mentioned above, for the case of low intrinsic permeability a reference value of $k_{0}=$ $10^{-18} \mathrm{~m}^{2}$ is chosen. With this value, no simulation result can be obtained that fits the experimental data very well regardless of which value of $\beta$ is used. Only if $\beta$ is zero, the result of two-phase flow simulation is roughly close to some experimental data, but the Richards' simulation never gives reasonable results. This is demonstrated in Fig. 6 for the saturation profile. A value of $\beta=0$ implies that the intrinsic permeability for gas flow at $S^{g}=1$ is 
equivalent to the intrinsic permeability for liquid flow at $S^{l}=1$, and that the intrinsic permeability does not change with saturation (see Eqn. (29)). Fig. 6 shows that for the same input parameters, there is less drying in the case of the full two-phase flow calculation. This is because in the two-phase flow calculation the gas pressure goes up at the hot end and thereby suppresses more drying. In the Richards' approach, the gas pressure is assumed constant, meaning this phenomenon is not accounted for.

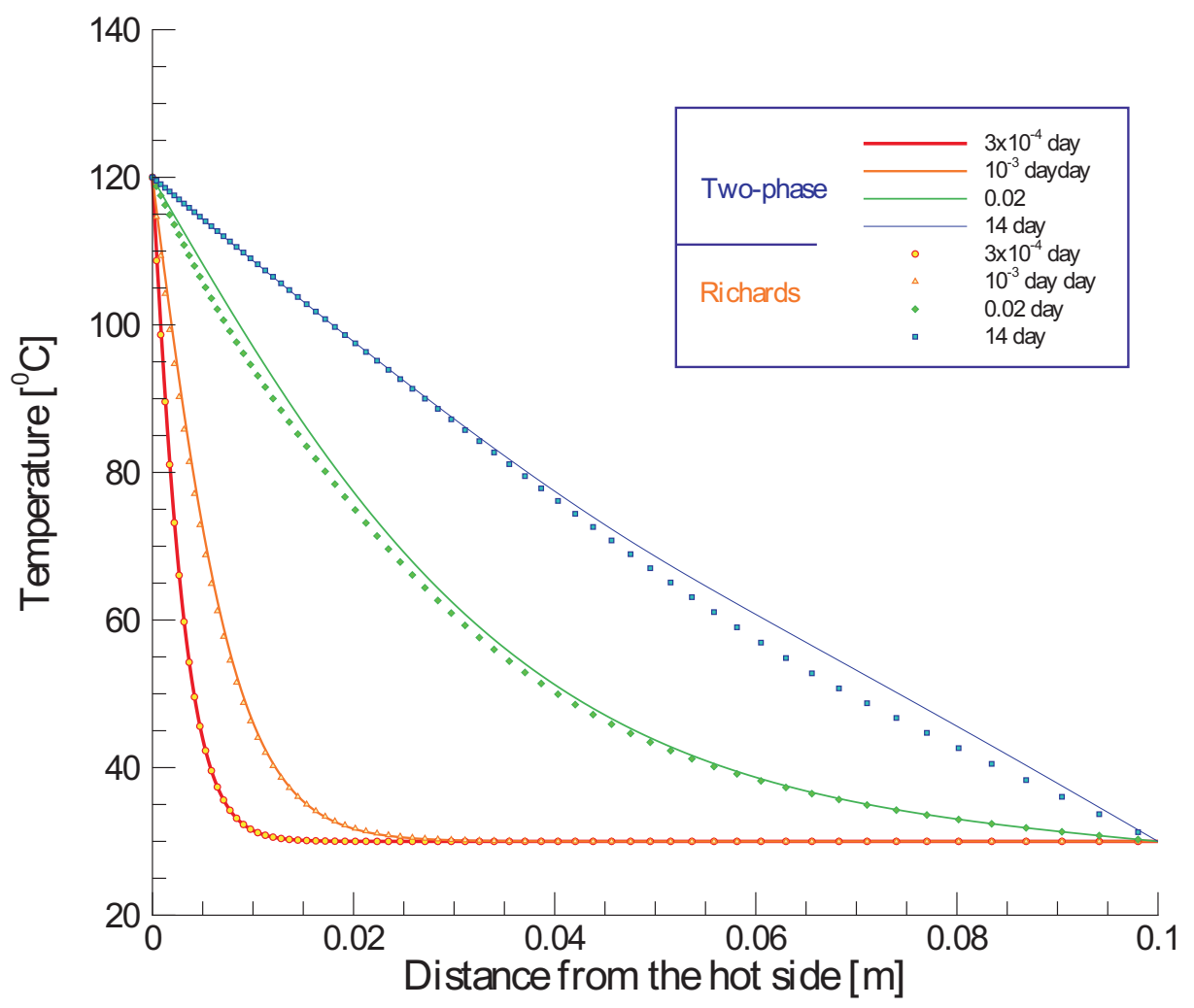

Fig. 7 Temperature profiles of CTF1 modeling with low intrinsic permeability at different times $t=3 \times 10^{-4}$, $0.1,0.5,14$ days

Contrary to saturation results, the temperature distributions in the domain obtained by the two-phase flow approach and the Richards' flow approach are similar as shown in Fig. 7 


\section{Ground surface}

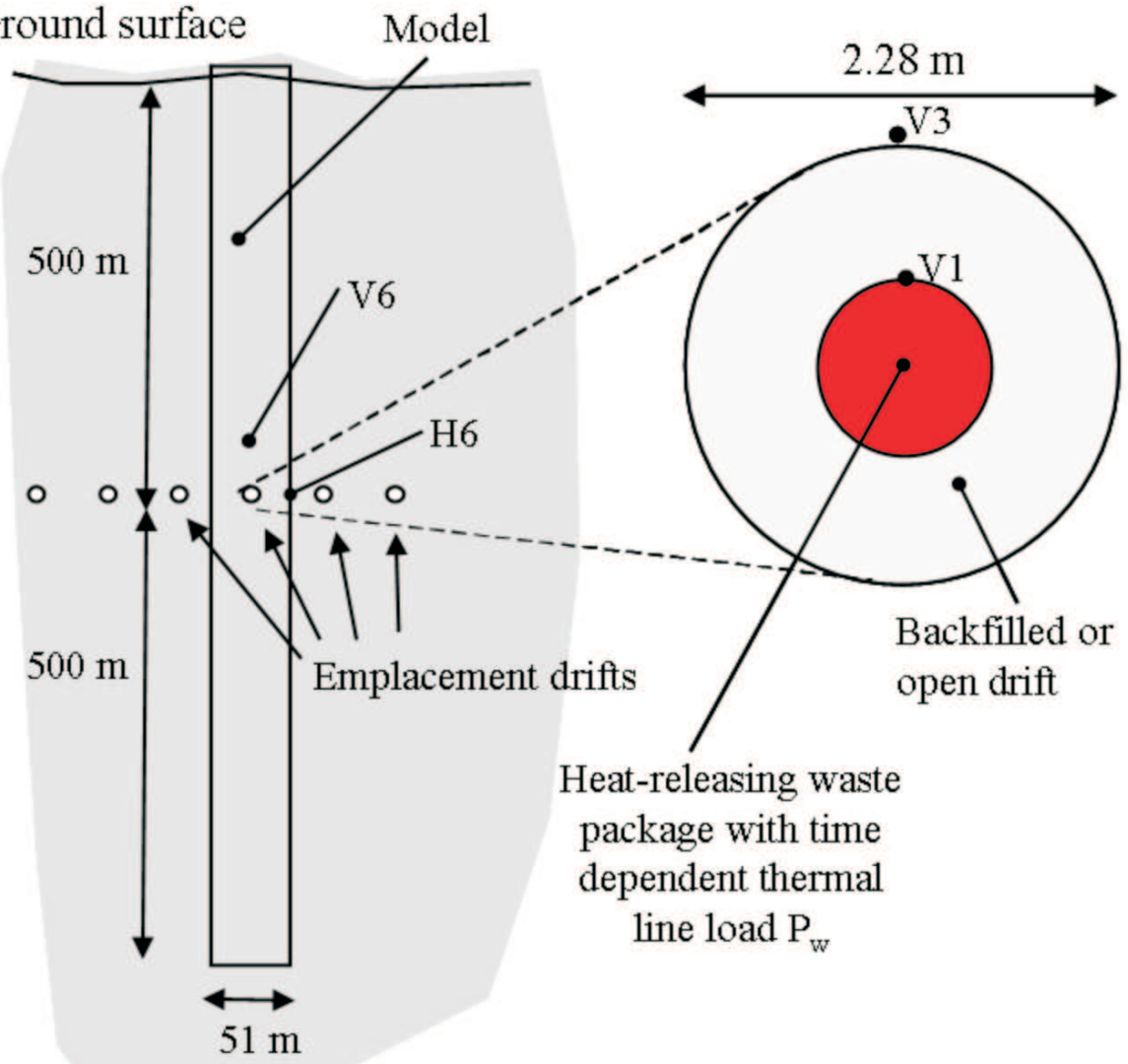

Fig. 8 Model domain for a benchmark test of a bentonite back-filled horizontal emplacement drift at $500 \mathrm{~m}$ (Rutqvist et al. 2009)

\subsection{Benchmark of longer-term TH processes around a nuclear waste repository}

As a second example, the longer-term TH processes occurring in the EBS of a geologic nuclear waste repository are considered by modeling a benchmark test that was originally part of the DECOVALEX-THMC project (Barr et al. 2004; Rutqvist et al. 2008, 2009). In the DECOVALEX-THMC project, this case was defined as FEBEX type of repository, because the geometry and material properties are based on those at the FEBEX in situ test conducted at the Grimsel Test Site in Switzerland. The geometry of the FEBEX type repository model 
considered in the DECOVALEX benchmark test is depicted in Fig. 8, where several specific

observation points for state variables are shown. Some of the key properties for the bentonite and rock are given in Tables 2 and 3 .

Table 2 Rock mass properties (Barr et al. 2004)

\begin{tabular}{lll}
\hline Parameter & Value & Unit \\
\hline Density & 2700.0 & $\mathrm{~kg} / \mathrm{m}^{3}$ \\
Porosity & 0.01 & - \\
Heat capacity & 900.0 & $\mathrm{~J} /(\mathrm{kg} \cdot \mathrm{K})$ \\
Heat conductivity & 3.0 & $\mathrm{~W} /(\mathrm{m} \cdot \mathrm{K})$ \\
Intrinsic permeability of liquid & $1.0 \times 10^{-17}$ & $\mathrm{~m}{ }^{2}$ \\
Intrinsic permeability of gas & $1.0 \times 10^{-17}$ & $\mathrm{~m}{ }^{2}$ \\
Air viscosity & $1.5 \times 10^{-5}$ & $\mathrm{~Pa} \cdot \mathrm{s}$ \\
Air density $\rho^{g}$ & Eqn. $(12)$ & \\
& with molar mass & \\
& of air & $\mathrm{kg} / \mathrm{m}^{3}$ \\
\hline
\end{tabular}

A time-depending heat power function is given in order to describe the heat source condition of the nuclear waste canister. The given heat source is converted into a heat flux boundary condition uniformly distributed on the boundary of the canister, and its evolution is depicted in Fig. 9.

Regarding the bentonite, in contrast to the very low intrinsic permeability of the liquid phase, the gas phase is assumed to have a higher saturation-dependent intrinsic permeability, which takes the form

$$
\mathbf{k} k_{\text {rel }}^{g}=5.695 \times 10^{-11}\left[e\left(1-S^{l}\right)\right]^{4.3} \mathbf{I}\left[\mathrm{m}^{2}\right]
$$


Table 3 Bentonite properties (Barr et al. 2004)

\begin{tabular}{lll}
\hline Parameter & Value & Unit \\
\hline Density & 1600.0 & $\mathrm{~kg} / \mathrm{m}^{3}$ \\
Tortuosity & 0.8 & - \\
Porosity & 0.41 & - \\
Heat capacity & $c_{s}=1.38 T+732.5$ & $\mathrm{~J} /(\mathrm{kg} \cdot \mathrm{K})$ \\
Heat conductivity & $\kappa_{m}=1.28-\frac{0.71}{1+e^{10\left(S^{l}-0.65\right)}}$ & $\mathrm{W} /(\mathrm{m} \cdot \mathrm{K})$ \\
Intrinsic permeability & & \\
of liquid & $2.0 \times 10^{-21}$ & $\mathrm{~m}{ }^{2}$ \\
Air viscosity & $1.5 \times 10^{-5}$ & $\mathrm{~Pa} \cdot \mathrm{s}$ \\
Air density $\rho^{g}$ & Eqn. $(12)$ & $\mathrm{kg} / \mathrm{m}^{3}$ \\
& with molar mass & \\
& of air &
\end{tabular}

with $e$, the void ratio, chosen to 1.44 . A detailed description of the model set-up can be found in (Wang et al. 2009).

Figs. 10 and 11 show the calculated evolution of temperature and saturation at selected output points V1 (in bentonite at canister surface), V3 (at bentonite-rock interface), V6 (in the rock $25 \mathrm{~m}$ from the canister). The GeoSys results are compared with those calculated with two other codes ROCMAS (Rutqvist et al. 2001) and TOUGH-FLAC (Rutqvist et al. 2002). The ROCMAS and TOUGH-FLAC codes are using Richards' and full two-phase flow approaches, respectively. Consequently, a code-to-code comparison can be performed in addition to the comparison of the two alternative modeling approaches.

Finally, Figs. 10 and 11 show that the results of the different models and modeling approaches are very similar and there is no significant systematic difference between the Richards' and full two-phase flow models. Some of the small but observable differences in 


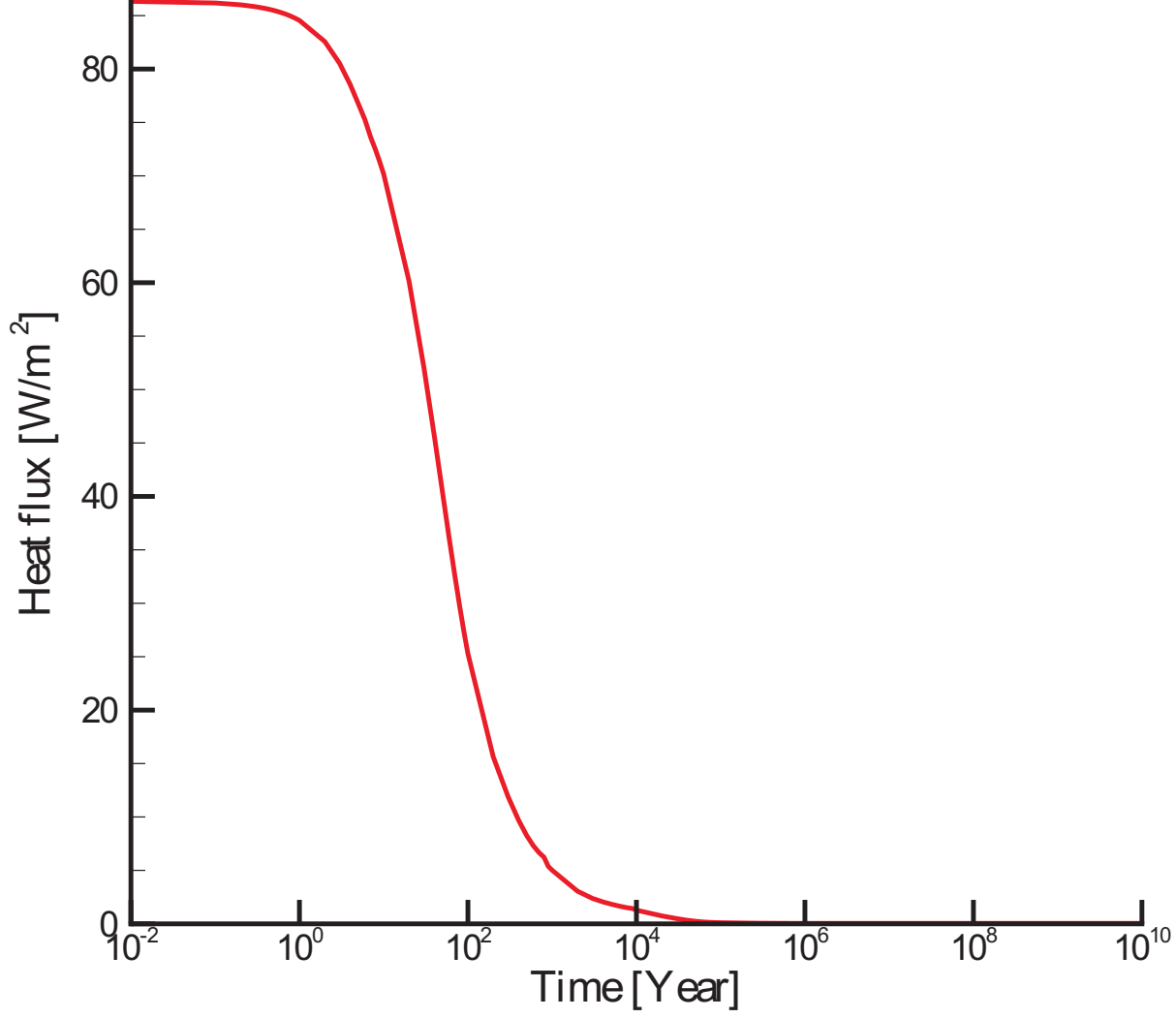

Fig. 9 Heat flux boundary condition on the canister surface (Barr et al. 2004; Rutqvist et al. 2009)

the results of the various models may be attributed to slight variations in the input properties for the bentonite, which may depend on the modeling approach used. Moreover, the way the codes interpolate the original heat output curve can also lead to some of the observed differences (cf. Rutqvist et al. 2009). However, the overall results indicate that both the Richards' and the full two-phase approaches are adequate in this case. The main reason is that the gas permeability is orders of magnitude higher than the liquid flow permeability. This is in agreement with the results obtained for the laboratory experiments, which indicated that both Richards' and two-phase flow models would be adequate when intrinsic permeability is relatively high. 
In the present study two common approaches for the simulation of non-isothermal flow processes in partially saturated porous media have been investigated: Richards' and two-phase flow model approaches. These approaches are widely used to study thermal-hydraulic processes in the near field of heat emitting waste deposited in geological formations including the impact on buffer materials such as bentonite for waste isolation. In this context, the understanding of vaporization phenomena due to increasing temperatures in low permeability porous media is important in order to unveil the influence of gas phase behavior on drying processes. The difference in the two approaches lies in the treatment of the gaseous phase. Two-phase models include the dynamic behavior of both fluid phases whereas in the Richards' model the gaseous phase is a passive (static) spectator.

For the comparison of the two approaches initially literature data from a laboratory experiment involving thermally driven moisture flow in a partially saturated bentonite sample have been studied. This experiment was simulated for the case that both liquid and gas phases have the same intrinsic permeability. Following, a benchmark test of longer-term thermal-hydraulic behavior in the EBS of a geological nuclear waste repository has been analyzed, which was characterized by a high gas intrinsic permeability. The findings show that both models can be used to reproduce the vaporization process if the intrinsic permeability is relative high. If a thermal-hydraulic coupled problem has the same low intrinsic permeability for both liquid and gas phases, only the two-phase flow approach can provide fairly reasonable results. The presented work provides an in depth insight into the validity of different non-isothermal flow models which is an important prerequisite for environmental safety assessment studies. 
Acknowledgements The development of the numerical models was conducted in the framework of the international DECOVALEX project. The funding from the Federal Institute for Geosciences is highly acknowledged (Dr. Shao). This work is part of the PoF research initiative of the Helmholtz Association within the Environmental Engineering and Geothermal Technology programs. Funding from the Swedish Radiation Safety Authority (SSM) through the U.S. Department of Energy Contract No. DE-AC02-05CH11231 is greatly appreciated.

\section{References}

Alkan H, Müller W (2008) Approaches for modelling gas flow in clay formations as repository systems. Phys Chem Earth 33(Supplement 1):S260-S268

Alonso EE, Alcoverro J, Coste F et al. (2005) The FEBEX benchmark test: case definition and comparison of modelling approaches. Int J Rock Mech Min Sci 42:611-638

Barr D, Birkholzer JT, Rutqvist J, Sonnenthal E (2004) Draft description for DECOVALEX-THMC Task D: Long-term permeability/porosity changes in EDZ and near field, due to THM and THC processes in volcanic and Crystalline-bentonite systems. Technical report, Earth Sciences Division, Lawrence Berkeley National Laboratory, USA

Börgesson L (1985) Water flow and swelling pressure in non-saturated bentonite-based clay barriers. Eng Geol 21:229-237

Börgesson L, Chijimatsu M, Fujita T, Nguyen TS, Rutqvist J, Jing L (2001) Thermo-hydro-mechanical characterization of a bentonite-based buffer material by laboratory tests and numerical back analyses. Int $\mathrm{J}$ Rock Mech Min Sci 38:95-104

Bruel D (2002) Impact of induced thermal stresses during circulation tests in an engineered fractured geothermal reservoir: example of the Soultzsous-Forets European hot fractured rock geothermal project, RhineGraben, France. Geothermics 57:459-470

Chijimatsu M, Börgesson L, Fujita T, Jussila P, Nguyen TS, Rutqvist J, Jing L (2009) Model development and calibration for the coupled thermal, hydraulic and mechanical phenomena of the bentonite. Environ Geol 57:1255-1261

Freiboth S, Class H, Helmig R, Graf T, Ehlers W, Schwarz V, Vrettos Ch (2009) A model for multiphase flow and transport in porous media including a phenomenological approach to account for deformationa model concept and its validation within a code intercomparison study. Computat Geosci 13:281-300 
Gens A, Alonso EE (1992) Framework for the behaviour of unsaturated expansive clays. Can Geotech J

29:1013-1032

Gou R, Dixon D (2006) Thermohydromechanical simulations of the natural cooling stage of the Tunnel Sealing Experiment. Eng Geol 85:313-331

Gray WG, Hassanizadeh SM (1991) Unsaturated flow theory including interfacial phenomena. Water Resour Res 27:1855-1963

Jang WY, Aral MM (2009) Multiphase flow fields in in-situ air sparging and its effect on remediation. Transp Porous Media 76:99-119

Kohl T, Evans KF, Hopkirk RJ, Rybach L (1995) Coupled hydraulic, thermal and mechanical considerations for the simulation of hot dry rock reservoirs. Geothermics 24:345-359

Kolditz O., Diersch H-J (1993) Quasi steady-state strategy for numerical simulation of geothermal circulation processes in hot dry rock fracture. Int J Non Linear Mech 28:467-481

Kolditz O, De Jonge JD (2004) Non-isothermal two-phase flow in low-permeable porous media. Comput Mech 33:345-364

Kuhn M, Bartels J, Iffland J (2002) Predicting reservoir property trends under heat exploitation: interaction between flow, heat transfer, transport, and chemical reactions in a deep aquifer at Stralsund, Germany. Geothermics 31:725-749

Lewis RW, Schrefler BA (1998) The finite element method in the static and dynamic deformation and consolidation of porous media. Second Edition, Wiley, Chichester

Li Q, Wu Z, Bai Y, Yin X, Li X (2006) Thermo-hydro-mechanical modeling of $\mathrm{CO}_{2}$ sequestration system around fault environment. Pure Appl Geophys 163:2585-2593

Nguyen TS, Börgesson L, Chijimatsu M, Hernelind J, Jing L, Kobayashi A, Rutqvist J (2009) A case study on the influence of THM coupling on the near field safety of a spent fuel repository in sparsely fractured granite. Environ Geol 57:1239-1254

Olivella S, Gens A (2000) Vapour transport in low permeability unsaturated soil with capillary effects. Transp Porous Media 40:219-241

O’Sullivan MJ, Pruess K, Lippmann MJ (2001) State of the art of geothermal reservoir simulation. Geother$\operatorname{mics} 30: 395-429$

Philip JR, de Vries DA (1957) Moisture movement in porous materials under temperature gradient. Trans Amer Geophys Union 38:222-232 
Pruess $\mathrm{K}$ (2008) $\mathrm{On} \mathrm{CO}_{2}$ fluid flow and heat transfer behavior in the subsurface, following leakage from a geologic storage reservoir. Environ Geol 54:1677-1686

Rutqvist J, Börgesson L, Chijimatsu M, Kobayashi A, Nguyen TS, Jing L, Noorishad J, Tsang CF (2001) Thermohydromechanics of partially saturated geological media - Governing equations and formulation of four finite element models. Int J Rock Mech Min Sci 38:105-127

Rutqvist J, Wu Y-S, Tsang C-F, Bodvarsson G (2002) A modeling approach for analysis of coupled multiphase fluid flow, heat transfer, and deformation in fractured porous rock. Int J Rock Mech Min Sci $39: 429-442$

Rutqvist J, Barr D, Birkholzer JT, Chijimatsu M, Kolditz O, Liu Q, Oda Y, Wang W, Zhang C (2008) Results from an international simulation study on coupled thermal, hydrological, and mechanical processes near geological nuclear waste repositories. Nucl Technol 163:101-109

Rutqvist J, Barr D, Birkholzer JT, Fujisaki K, Kolditz O, Liu Q, Fujita T, Wang W, Zhang C (2009) A comparative simulation study of coupled THM processes and their effect on fractured rock permeability around nuclear waste repositories. Environ Geol 57:1347-1360

Sanavia L, Pesavento F, Schrefler BA (2006) Finite element analysis of non-isothermal multiphase geomaterials with application to strain localization simulation. Comput Mech 37:331-348

Schrefler BA, Matteazzi R, Gawin D, Wang X (2000) Two parallel computing methods for coupled thermohydromechanical problems. Comput-Aided Civ Infrastruct Eng 15:176-188

Tsang C (1991) Coupled hydromechanical-thermochemical processes in rock fractures. Rev Geophys 29:537-551

van Genuchten MT (1980) A closed-form equation for predicting the hydraulic conductivity of unsaturated soils. Soil Sci Soc Am J 44:892-898

Villar MV, Fernandez AM, Cuevas J (1997) Caracterización Geoquímica de bentonita compactada: efectos producidos por flujo termohidráulico. Interim Report FEBEX, Informe 70-IMA-M-0-2, CIEMAT, Madrid

Wang W., Kosakowski G., Kolditz O. (2009) A parallel finite element scheme for thermo-hydro-mechanical (THM) coupled problems in porous media. Comput Geosci 35:1631-1641

Zhang XY, Zhu YM, Fang CH (2009) The role for air flow in soil slope stability analysis. J Hydrodyn 21:640646 


\section{List of Figure Captions}

1. Figure 1: Numerical model of the CTF1 experiment

2. Figure 2: Intrinsic permeability profiles in the simulations of CTF1 at different times $t=3 \times 10^{-4}, 0.1,0.5,14$ days: a) $k=10^{-13} \mathrm{~m}^{2}, \beta=4.2$; b) $k=10^{-11} \mathrm{~m}^{2}, \beta=7$

3. Figure 3: Saturation profiles by two-phase flow simulations of CTF1 at different times $t=3 \times 10^{-4}, 0.1,0.5,14$ days (experimental data is given for $t=14$ days)

4. Figure 4: Saturation profiles resulting from the simulations of CTF1 at different times $t=3 \times 10^{-4}, 0.1,0.5,14$ days: a) $k=10^{-13} \mathrm{~m}^{2}, \beta=4.2$; b) $k=10^{-11} \mathrm{~m}^{2}, \beta=7$ (experimental data is given for $t=14$ days)

5. Figure 5: Temperature profiles resulting from the simulations of CTF1 at different times $t=3 \times 10^{-4}, 0.1,0.5,14$ days: a) $k=10^{-13} \mathrm{~m}^{2}, \beta=4.2$; b) $k=10^{-11} \mathrm{~m}^{2}, \beta=7$

6. Figure 6: Saturation profiles of CTF1 modeling with low intrinsic permeability at different times $t=3 \times 10^{-4}, 0.1,0.5,14$ days (experimental data is given for $t=14$ days)

7. Figure 7: Temperature profiles of CTF1 modeling with low intrinsic permeability at different times $t=3 \times 10^{-4}, 0.1,0.5,14$ days

8. Figure 8: Model domain for a benchmark test of a bentonite back-filled horizontal emplacement drift at $500 \mathrm{~m}$ (Rutqvist et al. 2009)

9. Figure 9: Heat flux boundary condition on the canister surface (Barr et al. 2004; Rutqvist et al. 2009)

10. Figure 10: Temperature variation at points V1, V3 and V6

11. Figure 11: Saturation variation at point V1 